\title{
悬浮随机行走电容提取中多介质格林函数表的快速生成
}

\author{
杨明 1,2$)$, 喻文健 1,2$)^{*}$ \\ 1) (清华大学计算机科学与技术系 北京 100084) \\ 2) (北京信息科学与技术国家研究中心 北京 100084) \\ (yu-wj@tsinghua.edu.cn)
}

\begin{abstract}
摘 要：为加速悬浮随机行走预刻画, 利用了多介质格林函数本身的对称性, 通过增加虚拟齐次纽曼边界, 将有限 差分空间离散区域缩减至原本的 $1 / 4$, 从而显著减小了差分矩阵的规模, 加速了多介质格林函数表的生成. 一般多介 质格林函数表的对称性在理论上得到了证明. 所提出的数值计算方法基于 C++编程实现，其效率和正确性在一台 32 核服务器上通过数值实验得到了验证. 实验结果表明, 文中快速生成方法在达到超过 8 倍加速比的同时, 取得了与原 始有限差分方法结果完全一致的多介质格林函数表，显著减少了悬浮随机行走电容提取方法预处理的时间.
\end{abstract}

关键词：悬浮随机行走; 格林函数表; 有限差分; 电容提取; 狄利克雷问题

中图法分类号: TP391.41 DOI: 10.3724/SP.J.1089.2021.18508

\section{Accelerated Generation of Multi-Dielectric Green’s Function Table}

\author{
Yang Ming ${ }^{1,2)}$ and Yu Wenjian ${ }^{1,2)^{*}}$ \\ 1) (Department of Computer Science and Technology, Tsinghua University, Beijing 100084) \\ 2) (Beijing National Research Center for Information Science and Technology, Beijing 100084)
}

\begin{abstract}
A new method for fast generation of multi-dielectric Green's function table is proposed. The new method utilizes the symmetric property of multi-dielectric Green's function table. By inserting virtual homogeneous Neumann boundary inside the transition cube, the original cube with FDM grid imposed is cut down to one fourth of itself, which leads to smaller FDM matrices and faster generation of Green's function tables. The symmetry of multi-dielectric Green's function table is proved in theory and furthermore a new method for its fast computation is proposed who is implemented in C++ and whose efficiency and accuracy are validated through numerical experiments on a server with 32 cores. The experimental results show that the proposed method brings more than 8X speed-up compared to original FDM. Meanwhile, the Green function tables obtained are just as same as the original FDM. With the proposed method, pre-characterization time cost can be significantly reduced in FRW based capacitance extraction for multi-dielectric VLSI structures.
\end{abstract}

Key words: floating random walk; Green's function table; finite difference method; capacitance extraction; Dirichlet's problem

随着超大规模集成电路工艺的发展，芯片的 设计与制造已步入纳米时代. 晶体管的体积急剧
缩小, 其集成度按摩尔定律在一定时期内呈指数 增长，但同时高度集成的芯片也不得不直面纳米

收稿日期: 2020-07-22; 修回日期: 2020-09-29. 基金项目：国家自然科学基金(61872206); 北京市科技计划(Z201100004220003); 北京信息科学与技术国家研究中心课题(BNR2019ZS01001). 杨明(1995一), 男, 博士研究生, CCF 会员, 主要研究方向为大规模集 成电路仿真与建模、计算电磁学数值算法、电子设计自动化中机器学习的应用; 喻文健(1977一), 男, 博士, 副教授, 博士生导师, CCF 高级会员, 论文通讯作者, 主要研究方向为集成电路计算机辅助设计算法、机器学习与大数据分析算法、数值计算及其应用. 
节点下器件、互连线间寄生电容效应带来的挑战 ${ }^{[1]}$. 寄生电容不仅会带来更长的延迟，压低芯片的工 作频率, 还会导致额外能耗增加以及信号失真. 为 了应对这些挑战，大规模集成电路的寄生电容提 取成为了一项重要课题. 根据场求解器提取的寄 生参数, 芯片设计师可以针对寄生效应调整作品 设计, 从而提高其可靠性、信号完整性并降低能耗, 达到更高的流片成品率.

传统的电容提取方法包括有限差分、有限元以 及边界元等 ${ }^{[1]}$. 这些方法都需要对求解问题进行空 间离散化处理并求解线性方程组系统. 与这些传 统方法不同, 悬浮随机行走采用蒙特.卡罗法 ${ }^{[2]}$, 通过大量重复采样对求解结果进行准确估计, 无 需离散化处理, 也不必进行矩阵解算. 悬浮随机行 走法因为其低内存使用量、对大规模导体系统的扩 展性、可调节的求解精度以及易于并行的特点 ${ }^{[3]}$, 在工业界备受青睐 ${ }^{[4]}$. 学术界也研发了基于悬浮随 机行走的电容提取软件 $\mathrm{RWCap}^{[3,5]}$. 为将其扩展到 大规模复杂结构 ${ }^{[6-10]}$, 提高其并行处理能力 ${ }^{[11-13]}$, 相关学者也进行了大量的研究.

悬浮随机行走依赖于 3 项关键技术 ${ }^{[6-7]}$. 首先 是空间管理技术 ${ }^{[6]}$, 它可以大大加速悬浮随机行走 过程中的几何运算，从而显著提升算法效率. 其次 是方差削减技术 ${ }^{[6]}$, 它可以让蒙特. 卡罗法在更少 的随机行走数下收玫. 最后是预刻画技术 ${ }^{[7]}$, 它采 用有限差分, 为悬浮随机行走过程预备跳转使用 的采样概率表(格林函数表). 其中, 跨多层的多介 质格林函数表使悬浮随机行走过程中转移立方体 区域显著扩大, 大大降低随机行走的跳转次数, 加 速求解过程 ${ }^{[7]}$. 但是多介质格林函数表的生成需要 考虑介质交界面的所有可能的分布情况 ${ }^{[3,7]}$, 表的 建立过程耗时较长.

通过对大量多介质格林函数表的观察发现, 其具有几何对称性. 本文完成了相关证明，并提出 了一种利用该对称性的多介质格林函数表快速生 成法. 该方法的主要思路是在有限差分求解区域 内设置虚拟齐次纽曼边界 ${ }^{[14]}$, 将求解区域缩减为 原来的 $1 / 4$; 进而降低了有限差分矩阵的规模, 达 到加速多介质格林函数表生成的目的.

\section{1 相关工作}

\section{1 悬浮随机行走电容提取方法}

悬浮随机行走电容提取的原理是基于静电场 电势积分方程 ${ }^{[2-3]}$

$$
U(\boldsymbol{r})=\oiint_{S} P\left(\boldsymbol{r}, \boldsymbol{r}^{(1)}\right) U\left(\boldsymbol{r}^{(1)}\right) \mathrm{d} \boldsymbol{r}^{(1)}
$$

其中， $U(\boldsymbol{r})$ 代表空间中一点 $\boldsymbol{r}$ 处的电势; $S$ 代表 包围 $\boldsymbol{r}$ 点的闭合曲面; $P\left(\boldsymbol{r}, \boldsymbol{r}^{(1)}\right)$ 代表定义在 $S$ 上的 表面格林函数. 如图 1 所示, 其中黄色区域代表导 体, 取左下角导体作为主导体, 其周围蓝色虚线代 表高斯面. 随机行走首先在高斯面上采样得到起 点 $\boldsymbol{r}$, 继而以 $\boldsymbol{r}$ 为中心建立转移立方体 $T$, 持续跳 转直到采样点具有确定的电势, 如落在导体表面. 在实际悬浮随机行走过程中, $S$ 是以 $\boldsymbol{r}$ 为中心的转 移立方体 $T$ 的表面, 且要求 $T$ 不包含任何导体. 由 式(1)可知, $U(\boldsymbol{r})$ 可以看做对 $T$ 表面电势 $U\left(\boldsymbol{r}^{(1)}\right)$ 的一个随机抽样, 采样依据的概率分布函数即格 林函数. 这是因为从静电场理论可以验证表面格 林函数 $P$ 在其定义域 $S$ 上为非负函数且积分为 1 , 满足概率分布函数的数学定义 ${ }^{[2]}$. 为了估计 $U(\boldsymbol{r})$, 构造内无导体的转移立方体 $T$ 并依据格林函数 $P$ 在其表面 $S$ 上采样得到 $U\left(\boldsymbol{r}^{(1)}\right)$. 如果 $U\left(\boldsymbol{r}^{(1)}\right)$ 的电 势仍然未知, 则以 $\boldsymbol{r}^{(1)}$ 为新的转移立方体中心点继 续上述过程, 直到新的采样具有给定的电势 (如采 样点落在导体表面). 以上空间转移过程就是一次悬 浮随机行走; 通过进行成千上万次悬浮随机行走, 蒙特. 卡罗法能够对未知电势 $U(\boldsymbol{r})$ 给出精确估计.

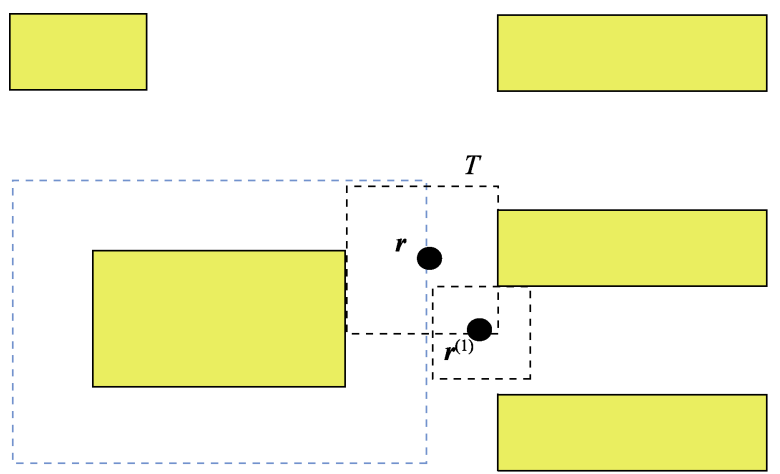

图 1 悬浮随机行走电容提取横截面示意图

电容提取需要算出主导体与其他导体的耦合 电容. 为了将电势计算与电容计算相关联, 主导体 周围建立起了一层闭合高斯面. 开始悬浮随机行 走时, 在这层高斯面上选定一点 $\boldsymbol{r}$ 建立第 1 个转移 立方体并得到采样点 $\boldsymbol{r}^{(1)}$. 由 $\boldsymbol{r}$ 与 $\boldsymbol{r}^{(1)}$ 可以确定一个 权重值, 其物理意义是对主导体与本次随机行走 结束时抵达的导体间耦合电容的估计. 当进行成 千上万次悬浮随机行走后, 这些权重值的均值就 构成了主导体和其他导体耦合电容的蒙特.卡罗估 计. 这个估计的误差可以通过设置随机行走数来 自由调节。 


\section{2 有限差分生成多介质格林函数表}

由第 1.1 节可知, 每次随机行走由一连串采样 跳转构成. 每次跳转起始于自转移立方体 $T$ 中心, 终止于表面 $S$ 上随机一点. 跳转所依据的概率转 移函数即为格林函数 $P$. 在实际应用中, 格林函数 $P$ 由有限差分法算得 ${ }^{[3,7]}$. 将转移立方体 $T$ 表面 $S$ 均匀分割成 $6 n^{2}$ 块面片, 并在 $T$ 内部和介质交界面 上施加差分网格, 即可通过差分矩阵运算得到格 林函数表. 格林函数表的 $6 n^{2}$ 个表项代表从 $T$ 中心 转移到各个面片中心的概率. 式(1)据此被改写为 离散的形式

$$
U(\boldsymbol{r})=\sum_{i=1}^{6 n^{2}} P_{i} U\left(\boldsymbol{r}_{i}\right)
$$

其中， $U\left(\boldsymbol{r}_{i}\right)$ 代表第 $i$ 个面片中心点的电势; $P_{i}$ 代 表其对应的格林函数表表项.

差分法网格中的网格点可以按所处位置分为 3 类, 依次为内部点、边界点和交界面点 ${ }^{[3]}$. 如图 2 所示, 图 2a 中对内部点 $u_{0}$ 处二阶偏导数进行有限 差分近似, 其中, $u_{1}$ 和 $u_{-1}$ 为其相邻网格点的电势, $h$ 为内部网格间距. 图 $2 \mathrm{~b}$ 中对交界面点 $u_{0}$ 处二阶 偏导数进行有限差分近似，其中橘黄色虚线代表 介质交界面. 图 2c 中通过对与转移立方体中心点 最邻近的 8 个网格点电势 $u_{1} \sim u_{8}$ 插值近似计算中心 点处电势 $u_{\mathrm{C}}$. 对于内部点, 其满足拉普拉斯方程

$$
\Delta U(\boldsymbol{r})=0
$$

应用中心差分公式

$$
\frac{\partial^{2} U(\boldsymbol{r})}{\partial^{2} x^{2}} \approx \frac{u_{1}-2 u_{0}+u_{-1}}{h^{2}}
$$

近似计算二阶导数, 如图 2a 所示. 对于边界点, 因 为转移立方体表面可以视为狄利克雷边界 ${ }^{[14]}$, 无 需差分近似. 对于交界面点, 因为处于不同介质交 界面上，满足交界面条件

$$
\varepsilon^{+} \frac{\partial U(\boldsymbol{r})}{\partial z^{+}}=\varepsilon^{-} \frac{\partial U(\boldsymbol{r})}{\partial z^{-}}
$$

为提高求解精度，应用二阶差分公式近似计算沿 交界面法向量的电场分量，式(5)近似为

$$
\varepsilon^{+} \frac{8 u_{1}-9 u_{0}+u_{2}}{6 h}=\varepsilon^{-} \frac{-8 u_{-1}+9 u_{0}-u_{-2}}{6 h}
$$

如图 2b 所示. 如果将网格点编号, 使任一内部点的 序号皆小于边界点的序号, 任一边界点的序号皆小 于交界面点序号, 则可以得到差分方程组 ${ }^{[3]}$

$$
\left[\begin{array}{rrr}
\boldsymbol{E}_{11} & \boldsymbol{E}_{12} & \boldsymbol{E}_{13} \\
\mathbf{0} & \boldsymbol{I}_{2} & \mathbf{0} \\
\boldsymbol{E}_{31} & \mathbf{0} & \boldsymbol{E}_{33}
\end{array}\right]\left[\begin{array}{l}
\boldsymbol{u}_{\mathrm{I}} \\
\boldsymbol{u}_{\mathrm{B}} \\
\boldsymbol{u}_{\mathrm{F}}
\end{array}\right]=\left[\begin{array}{l}
\mathbf{0}_{\mathrm{C}} \\
\boldsymbol{v}_{\mathrm{B}} \\
\mathbf{0}_{\mathrm{C}}
\end{array}\right]
$$

其中, $\boldsymbol{u}_{\mathrm{I}}, \boldsymbol{u}_{\mathrm{B}}, \boldsymbol{u}_{\mathrm{F}}$ 分别代表内部点、边界点和交界面 点的电势; $v_{\mathrm{B}}$ 代表施加在转移立方体表面的狄利 克雷边界条件; 0 代表全零列向量; $\mathbf{0}_{\mathrm{C}}$ 代表全零 矩阵; $\boldsymbol{I}_{2}$ 是一个单位方阵. 易知 $\boldsymbol{E}$ 作为有限差分 系数矩阵, 是一个稀疏矩阵. 如果将格林函数表记 为列向量 $\boldsymbol{P}_{\mathrm{k}}$, 则由式(7)可导出 ${ }^{[3]}$, 即

$$
\boldsymbol{P}_{\mathrm{k}}=-\left(\left(\boldsymbol{E}_{11}-\boldsymbol{E}_{13} \boldsymbol{E}_{33}^{-1} \boldsymbol{E}_{31}\right)^{-\mathrm{T}} \boldsymbol{e}_{\mathrm{k}}\right)^{\mathrm{T}} \boldsymbol{E}_{12}
$$

其中, $e_{\mathrm{k}}$ 为一个稀疏列向量, 其维度等于内部网 格点总数. 它仅有的 8 个非零元代表利用转移立方 体中心附近紧邻的 8 个内部网格点生成格林函数 表的插值系数. 其对应对插值公式为

$$
u_{\mathrm{C}}=\frac{\varepsilon^{+}\left(u_{1}+u_{2}+u_{3}+u_{4}\right)+\varepsilon^{-}\left(u_{5}+u_{6}+u_{7}+u_{8}\right)}{4\left(\varepsilon^{+}+\varepsilon^{-}\right)}
$$

其中, $u_{\mathrm{C}}$ 代表转移立方体中心电势, 如图 2c 所示. 在预刻画阶段, 需要生成悬浮随机行走过程中使 用的格林函数表. 若允许转移立方体跨越多层介 质, 就能够显著地减少每次随机行走的跳转次数 ${ }^{[7]}$, 但相应地就要计算所有可能的多介质格林函数表. 假设允许转移立方体跨越 3 层介质, 同时将转移立 方体 3 个方向的表等距划分为 32 份进行有限差分, 那么 2 个介质交界面有可能出现在 $z$ 方向上的 31 处差分位置. 针对一组给定 3 层介电常数配比, 需 要生成 $(31 \times 30) / 2=465$ 张多介质格林函数表 ${ }^{[3]}$. 虽 然这些多介质格林函数表可以重复利用, 但在预刻 画阶段是很大的计算量, 存在进一步优化的空间. 本文利用了多介质格林函数表本身的对称性，通过 在转移立方体中插人虚拟齐次纽曼边界, 将求解区 域缩减为原来的 $1 / 4$, 进而降低了有限差分矩阵的规 模, 最终达到加速多介质格林函数表生成的效果.

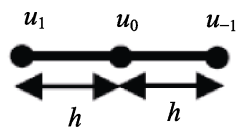

a. 中心差分图

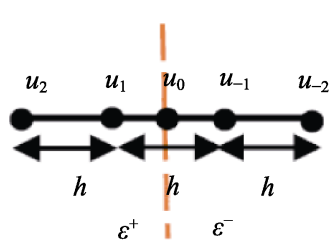

b. 交界面处差分图

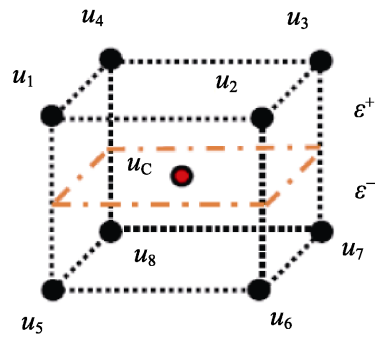

c. 中心点网格近似图
图 2 预刻画阶段有限差分公式示意图 


\section{2 多介质格林函数表的对称性证明与快速 生成}

\section{1 多介质格林函数表的对称性}

如图 3 所示, 图 3a 展示了格林函数表关于纵 截面的对称性. 转移立方体 $T$ 的中心为 $\boldsymbol{r}$, 表面为 $S$. 转移立方体的 2 个纵截面分别记为 $H$ 和 $H^{\prime}$. $\boldsymbol{r}^{(1)}$ 和 $\boldsymbol{r}^{(2)}$ 为 $S$ 上关于 $H$ 呈镜面对称的 2 点. 图 3b 中 $H$ 和 $H^{\prime}$ 分割出的子区域 $T_{\mathrm{p}}$ 的俯视图, 阴影区 域代表其外表面 $S_{\mathrm{p}}$. 记转移立方体 $T$ 的中心为 $\boldsymbol{r}$, 取其表面上 $S$ 任一点 $\boldsymbol{r}^{(1)}$, 再取与 $\boldsymbol{r}^{(1)}$ 关于过 $\boldsymbol{r}$ 的纵 截面 $H$ 镜面对称的另一点 $\boldsymbol{r}^{(2)}$, 则基于静电场理 论，有等式

$$
P\left(\boldsymbol{r}, \boldsymbol{r}^{(1)}\right)=P\left(\boldsymbol{r}, \boldsymbol{r}^{(2)}\right)
$$

成立.

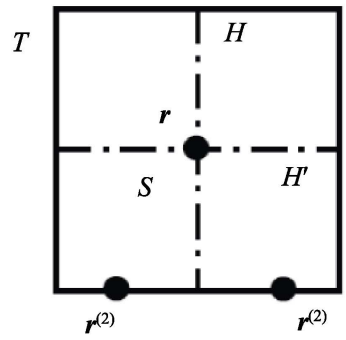

a. 原始有限差分区域

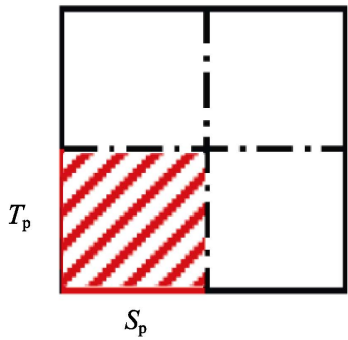

b. 削减后的有限差分区域
图 3 多介质格林函数表的对称性俯视示意图

为理解格林函数的对称性, 对原转移立方体 关于图 3a 中的纵截面 $H$ 作镜面反射变换, 得到的 新转移立方体 $T^{\prime}$. 显然对于包含多层介质的转移 立方体 $T, T^{\prime}$ 与 $T$ 完全相同. 因此, 二者的格林函 数在转移立方体表面 $S$ 上任一点 $\boldsymbol{r}^{(1)}$ 均有 $P^{\prime}\left(\boldsymbol{r}, \boldsymbol{r}^{(1)}\right)=P\left(\boldsymbol{r}, \boldsymbol{r}^{(1)}\right)$. 但是同时，因为 $T^{\prime}$ 与 $T$ 又关 于纵截面 $H$ 互为镜像, 对 $\boldsymbol{r}^{(1)}$ 与其镜像点 $\boldsymbol{r}^{(2)}$, $P^{\prime}\left(\boldsymbol{r}, \boldsymbol{r}^{(1)}\right)=P\left(\boldsymbol{r}, \boldsymbol{r}^{(2)}\right)$. 综合上述等价关系, 即得出 式(10).

另外, 对于多介质格林函数表 $\boldsymbol{P}_{\mathrm{k}}$, 同样考虑 转移立方体 $T$ 关于 $H$ 作镜面反射变换后得到的新 转移立方体 $T^{\prime}$, 对 $T^{\prime}$ 应用有限差分可生成镜像多 介质格林函数表 $\boldsymbol{P}_{\mathrm{k}}^{\prime}$. 从矩阵的角度, 镜面反射变 换相当于对第 1.2 节差分矩阵 $\boldsymbol{E}$ 进行了行列重排 序(详见后续的证明过程), 其对应于一排列阵

$$
\boldsymbol{M}=\left[\begin{array}{rrr}
\boldsymbol{M}_{\mathrm{I}} & \mathbf{0} & \mathbf{0} \\
\mathbf{0} & \boldsymbol{M}_{\mathrm{B}} & \mathbf{0} \\
\mathbf{0} & \mathbf{0} & \boldsymbol{M}_{\mathrm{F}}
\end{array}\right]
$$

其中, $\boldsymbol{M}_{\mathrm{I}}, \boldsymbol{M}_{\mathrm{B}}, \boldsymbol{M}_{\mathrm{F}}$ 矩阵分别代表转移立方体 $T$ 关 于 $H$ 作镜面反射变换而导致内部网格点、边界网 格点以及交界面网格点序号重排的对应排列阵. 从矩阵角度重述式(10), 得到 $\boldsymbol{P}_{\mathrm{k}}$ 的性质为

$$
\boldsymbol{P}_{\mathrm{k}}=\boldsymbol{M}_{\mathrm{B}} \boldsymbol{P}_{\mathrm{k}}
$$

虽然从物理意义上讲, 格林函数本身具有镜 面对称性, 但是应用特定数值算法生成的多介质 格林函数表能否同样具有式(12)所描述的对称性, 需要进一步确认. 本文将证明在第 1.2 节描述的数 值计算框架下, 式(12)对其生成的多介质格林函数 表 $\boldsymbol{P}_{\mathrm{k}}$ 严格成立.

首先考查第 1.2 节描述的有限差分法所建立的 差分网格 $G$. 因为该网格是等间距标准差分网格, 并且施加该网格的转移立方体区域 $T$ 本身关于纵 截面 $H$ 具有镜面对称性. 所以对 $G$ 作镜面反射变 换得到的镜像差分网格 $G^{\prime}$ 与 $G$ 本身在空间上重合, 它们唯一的差异是各网格点的序号- $G^{\prime}$ 中任一 网格点的序号等于其反射变换原像网格点在 $G$ 中 的编号. 由此, 可以根据这一序号重排法则定义式 (12)中的排列阵 $\boldsymbol{M}$. 同时, 考查内部网格点与交 界面网格点处应用式(4)(6), 它们的差分系数不受 镜面反射变换的影响. 具体而言, 镜面反射变换后 的差分矩阵为

$$
\boldsymbol{E}^{\prime}=\left[\begin{array}{rrr}
\boldsymbol{M}_{\mathrm{I}} \boldsymbol{E}_{11} \boldsymbol{M}_{\mathrm{I}}^{\mathrm{T}} & \boldsymbol{M}_{\mathrm{I}} \boldsymbol{E}_{12} \boldsymbol{M}_{\mathrm{B}}^{\mathrm{T}} & \boldsymbol{M}_{\mathrm{I}} \boldsymbol{E}_{13} \boldsymbol{M}_{\mathrm{F}}^{\mathrm{T}} \\
\mathbf{0} & \boldsymbol{I}_{2} & \mathbf{0} \\
\boldsymbol{M}_{\mathrm{F}} \boldsymbol{E}_{31} \boldsymbol{M}_{\mathrm{I}}^{\mathrm{T}} & \mathbf{0} & \boldsymbol{M}_{\mathrm{F}} \boldsymbol{E}_{33} \boldsymbol{M}_{\mathrm{F}}^{\mathrm{T}}
\end{array}\right]
$$

其中， $\boldsymbol{E}^{\prime}$ 代表基于网格 $G^{\prime}$ 建立的差分矩阵. 由此 可见, 镜面反射变换相当于对原差分矩阵进行了 行列重排. 而基于差分矩阵 $\boldsymbol{E}^{\prime}$ 与式(8)生成的多介 质格林函数 $\boldsymbol{P}_{\mathrm{k}}^{\prime}$ 为

$$
\boldsymbol{P}_{\mathrm{k}}^{\prime}=-\left(\left(\boldsymbol{E}_{11}^{\prime}-\boldsymbol{E}_{13}^{\prime} \boldsymbol{E}_{33}^{\prime-1} \boldsymbol{E}_{31}^{\prime}\right)^{-\mathrm{T}} \boldsymbol{e}_{\mathrm{k}}^{\prime}\right)^{\mathrm{T}} \boldsymbol{E}_{12}^{\prime}
$$

其中, $\boldsymbol{E}_{11}^{\prime}, \boldsymbol{E}_{12}^{\prime}, \boldsymbol{E}_{13}^{\prime}, \boldsymbol{E}_{31}^{\prime}$ 和 $\boldsymbol{E}_{33}^{\prime}$ 为式(13)中相应位置 的重排子矩阵; $\boldsymbol{e}_{\mathrm{k}}^{\prime}$ 为按照 $\boldsymbol{M}_{\mathrm{I}}$ 重排后的稀疏列向量. 而进一步考查 $\boldsymbol{E}^{\prime}$ 和 $\boldsymbol{E}$ 中非零元的关系, 会发现变 换前后的 2 个差分矩阵相同. 取 $\boldsymbol{E}^{\prime}$ 中任一非零元 $E_{i j}^{\prime}$, 它的含义是网格 $G^{\prime}$ 中编号为 $i$ 和 $j$ 的网格点 对应的差分系数. 再考虑 $\boldsymbol{E}$ 中具有相同下标的非 零元 $E_{i j}$, 因为网格 $G$ 中编号为 $i$ 和 $j$ 的网格点与 网格 $G^{\prime}$ 中编号为 $i$ 和 $j$ 的网格点关于纵截面 $H$ 镜 面对称, 易知其具有相同的差分系数, 所以 $E_{i j}$ 与 $E_{i j}^{\prime}$ 相等. 对于式(8)中的 $\boldsymbol{e}_{\mathrm{k}}$, 易证它本身就等于重 排后的 $M_{\mathrm{I}} \boldsymbol{e}_{\mathrm{k}}$, 所以 


$$
\boldsymbol{P}_{\mathrm{k}}^{\prime}=\boldsymbol{P}_{\mathrm{k}}
$$

另外, 因为 $\boldsymbol{P}_{\mathrm{k}}^{\prime}$ 是 $\boldsymbol{P}_{\mathrm{k}}$ 镜面反射变换的像, 故其 也是 $\boldsymbol{P}_{\mathrm{k}}$ 的重排, 即

$$
\boldsymbol{P}_{\mathrm{k}}^{\prime}=\boldsymbol{M}_{\mathrm{B}} \boldsymbol{P}_{\mathrm{k}}
$$

综合式(15)(16), 本文证明了式(12)严格成立.

注意, 若将纵截面 $H$ 旋转 $90^{\circ}$, 则得到多介质 格林函数表的另一个对称镜面 $H^{\prime}$, 如图 3a 所示. 多介质格林函数表也关于其对称. 相关证明与上 述过程完全一致, 故不在此赘述.

\section{2 多介质格林函数表快速生成}

由第 2.1 节可知, 多介质格林函数表具有镜面 对称性, 其对称平面为转移立方体 $T$ 的 2 个正交的 纵截面. 同时, 如第 1.2 节所述, 为含有多层介质 的转移立方体进行预刻画需要很大的计算量. 本 文探索利用多介质格林函数表的对称性设计其快 速生成方法, 以加速含有多层介质转移立方体的 预刻画过程.

使用有限差分法建立格林函数表的过程，与 求解静电场狄利克雷问题 ${ }^{\circledR}$ 有着密切的联系. 在狄 利克雷问题中, 给定求解的区域边界点的值, 则该 区域内部各点的值将被这组边界值唯一确定. 该 决定关系在式(1)中量化为表面格林函数. 格林函 数本身与边界电势的取值无关, 而是由求解区域 的几何特征以及内部介质分布确定. 利用这一点, 通过在转移立方体表面设置特殊的狄利克雷边界 条件, 即可在转移立方体 $T$ 内部 2 个正交纵截面上 形成齐次纽曼边界, 从而缩减求解区域, 加速多介 质格林函数表的生成. 如图 3a 所示, 对 $T$ 表面 $S$ 上任一点 $\boldsymbol{r}^{(1)}$ 和与之关于 $H$ 或 $H^{\prime}$ 镜面对称的另一 点 $\boldsymbol{r}^{(2)}$ ，设置具有对称性的边界条件

$$
U\left(\boldsymbol{r}^{(1)}\right)=U\left(\boldsymbol{r}^{(2)}\right)
$$

则由静电场理论不难证明 $T$ 内部对电场分布也是 关于 $H$ 或 $H^{\prime}$ 镜面对称的. 同时对于 $T$ 的中心 $\boldsymbol{r}$ 式 (8)变为

$$
U(\boldsymbol{r})=\iint_{S_{\mathrm{p}}} P_{\mathrm{p}}\left(\boldsymbol{r}, \boldsymbol{r}^{(1)}\right) U\left(\boldsymbol{r}^{(1)}\right) \mathrm{d} \boldsymbol{r}^{(1)}
$$

其中， $S_{\mathrm{p}}$ 表示利用对称性削减后 $T$ 的外表面，是 $S$ 的 1/4, 如图 3b 所示; $P_{\mathrm{p}}\left(\boldsymbol{r}, \boldsymbol{r}^{(1)}\right)$ 代表中心点电势 受到 $S_{\mathrm{p}}$ 边界上电势影响的程序. 由表面格林函数 的对称性可知, $P_{\mathrm{p}}\left(\boldsymbol{r}, \boldsymbol{r}^{(1)}\right)$ 与 $P\left(\boldsymbol{r}, \boldsymbol{r}^{(1)}\right)$ 的关系为

$$
P_{\mathrm{p}}\left(\boldsymbol{r}, \boldsymbol{r}^{(1)}\right)=4 P\left(\boldsymbol{r}, \boldsymbol{r}^{(1)}\right)
$$

但是 $P_{\mathrm{p}}\left(\boldsymbol{r}, \boldsymbol{r}^{(1)}\right)$ 的计算代价要比 $P\left(\boldsymbol{r}, \boldsymbol{r}^{(1)}\right)$ 小许多, 因为只需要在原问题的子区域 $T_{\mathrm{p}}$ 作有限差分, $H$ 与 $H^{\prime}$ 上的齐次纽曼边界会把 4 个子区域解耦分隔 开. 在计算 $P_{\mathrm{p}}\left(\boldsymbol{r}, \boldsymbol{r}^{(1)}\right)$ 时, 网格的划分策略可以完 全继承自第 2.2 节的方法, 将差分网格点按照子区 域 $T_{\mathrm{p}}$ 内部点、边界点和交接面点排序. 但是需要注 意的是, 子区域 $T_{\mathrm{p}}$ 有 2 类边界, 即与转移立方体 $T$ 表面重合的外边界 $S_{\mathrm{p}}$, 以及虚拟插人的齐次纽曼 边界. 本文方法只对前者进行编号, 对于后者, 只 需增加齐次纽曼边界条件

$$
u_{\mathrm{nb}}-u_{\mathrm{I}}=0
$$

即可在针对内部点列差分方程时消去. 其中, $u_{\mathrm{nb}}$ 代表齐次纽曼边界上的网格点电势; $u_{\mathrm{I}}$ 代表与之 相邻的内部网格点电势. 如此得到的规模缩减的 差分矩阵方程为

$$
\left[\begin{array}{rrr}
A_{11} & A_{12} & A_{13} \\
O & I_{2} & O \\
A_{31} & O & A_{33}
\end{array}\right]\left[\begin{array}{l}
u_{\mathrm{I}}^{*} \\
u_{\mathrm{B}}^{*} \\
u_{\mathrm{F}}^{*}
\end{array}\right]=\left[\begin{array}{r}
0 \\
v_{\mathrm{B}}^{*} \\
0
\end{array}\right]
$$

其分块的依据与第 1.2 节相同, $\boldsymbol{u}_{\mathrm{I}}^{*}, \boldsymbol{u}_{\mathrm{B}}^{*}, \boldsymbol{u}_{\mathrm{F}}^{*}$ 和 $\boldsymbol{v}_{\mathrm{B}}^{*}$ 代 表维度缩减后的电势列向量. 同时, 将式(8)中的 $\boldsymbol{e}_{\mathrm{k}}$ 限制在子区域 $T_{\mathrm{p}}$ 中得到 $\boldsymbol{e}_{\mathrm{k}}^{*}$, 其长度减小至 $1 / 4$, 非零元数目减少至 2 个. 该稀疏列向量的含义是, 使用子区域 $T_{\mathrm{p}}$ 中与 $T$ 的中心 $\boldsymbol{r}$ 最邻近的 2 个网格点 插值计算 $\boldsymbol{r}$ 处的多介质格林函数表. 其插值公式为

$$
u_{\mathrm{C}}=\frac{\varepsilon^{+} u_{1}+\varepsilon^{-} u_{5}}{\varepsilon^{+}+\varepsilon^{-}}
$$

该插值公式可由式(9)导出. 如图 2c 所示, 因为对 称边界条件的选取, $\boldsymbol{r}$ 上方 4 个网格点电势与 $u_{1}$ 相 同, $\boldsymbol{r}$ 下方 4 个网格点电势与 $u_{5}$ 相同. 结合式 (19)(21)(22), 得到新方法中多介质格林函数表的 计算式为

$$
\boldsymbol{P}_{\mathrm{k}}{ }^{*}=-\frac{1}{4}\left(\left(\boldsymbol{A}_{11}-\boldsymbol{A}_{13} \boldsymbol{A}_{33}^{-1} \boldsymbol{A}_{31}\right)^{-\mathrm{T}} \boldsymbol{e}_{\mathrm{k}}\right)^{\mathrm{T}} \boldsymbol{A}_{12}
$$

其中, $\boldsymbol{P}_{\mathrm{k}}{ }^{*}$ 对应 $1 / 4$ 边界 $S_{\mathrm{p}}$ 上多介质格林函数表的 表项, 将其按照镜面对称性复制扩充到原边界 $S$ 上即可得到 $\boldsymbol{P}_{\mathrm{k}}$. 至此, 本文利用格林函数表自身 的对称性, 通过对转移立方体施加对称边界条件, 在其内部构造出齐次纽曼边界, 将原问题差分求 解区域削减至 $1 / 4$, 有效地降低了差分矩阵的规模, 
从而提高了多介质格林函数表生成的效率, 减少 了预刻画阶段的内存和时间的开销.

\section{3 数值实验}

为了验证第 2 节所提方法的有效性和准确性, 本文设计了一组多介质格林函数表生成的对照实 验, 从耗时与准确度 2 个维度对数值实验结果的比 较. 结果表明, 本文方法显著缩减了生成多介质格 林函数表的时间开销，同时得到的结果与原始方 法完全一致. 所有实验都是在一台 32 核的服务器 上基于 $\mathrm{C}++$ 开发的程序 TechGFT ${ }^{[7]}$ 开展的. 该服务 器为 $\operatorname{Intel}(\mathrm{R}) \operatorname{Xeon}(\mathrm{R})$ Silver $4110 \mathrm{CPU}$, 主频为 $2.1 \mathrm{GHz}$. 多介质格林函数表建立过程中底层的向 量矩阵运算支持来自开源 BLAS 库 Openblas ${ }^{\circledR}$ 和稀 疏矩阵求解库 SuiteSparse ${ }^{[15]}$.

图 4 所示为一个包含 3 层介质的转移立方体的 数值实验的例子，虚线代表相邻 2 层介质交界面， 交界面分布仅为示意，实际会针对差分网格的设 置计算尽可能多的介质交界面分布情况. 3 层介质 的相对介电常数由下到上依次为 3.9, 6.5, 3.5. 下 面将分别从格林函数表的生成速度和结果准确度 评价新生成方法的有效性.

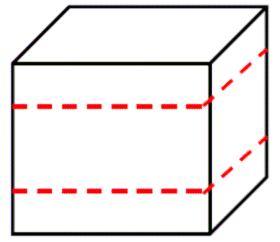

图 4 针对 3 层转移立方体建立格林函数表图例

\section{1 生成多介质格林函数表的速度提升验证}

从理论上分析，假如稀疏矩阵求解的时间复 杂度在本问题中严格保持为 $O\left(N^{1.5}\right)^{[15]}$, 其中, $N$ 为差分矩阵维度, 那么预期的加速比应为 8 , 因为 本文方法将差分区域缩小至原来的 $1 / 4$. 本实验针 对 3 层转移立方体的例子设置了不同的离散粒度 $n$ (32，40，48 和 56), 它代表立方体的每一条边被 有限差分网格均分的份数. 表 1 中记录了对于不同 $n$ 原始和改进的有限差分法多介质格林函数表的 生成时间. 从表 1 可以看出, 改进后的有限差分方 法比原有限差分方法实际的加速比是超过 8 的，这 是因为在转移立方体预刻画中差分矩阵求解的时 间复杂度可能会略微超过 $O\left(N^{1.5}\right)$ 的估计.
表 $1 n$ 不同时 2 种方法生成格林函数表耗时

\begin{tabular}{cccc}
\hline \multirow{2}{*}{$n$} & \multicolumn{2}{c}{ 有限差分耗时/s } & \multirow{2}{*}{ 加速比 } \\
\cline { 2 - 3 } & 原始 & 改进 & \\
\hline 32 & 192.8 & 23.2 & 8.3 \\
40 & 981.0 & 94.7 & 10.4 \\
48 & 3962.6 & 296.5 & 13.4 \\
56 & 9256.7 & 861.4 & 10.7 \\
\hline
\end{tabular}

\section{2 生成多介质格林函数表的准确度校验}

为了验证改进后有限差分法的准确性，本文 将 2 种方法建立的 3 层格林函数表进行了比较, 如 图 5 和图 6 所示. 观察图 5, 图 5a 为本文所提出的 改进有限差分法建立的局部格林函数表, 图 $5 \mathrm{~b}$ 为 原始有限差分方法建立的全局格林函数表. 可以 看出, 将图 5a 中的局部格林函数表对称地复制 4 份, 即可还原图 $5 \mathrm{~b}$ 中格林函数表. 其背后的理论 依据是格林函数表的对称性, 这一对称性也在图 $5 \mathrm{~b}$ 中得到了印证. 本实验还进一步具体比较了 2 种方法生成格林函数表的每一个表项, 如图 6 所 示, 图中横坐标代表本文提出改进有限差分法算 得的格林函数表表项, 纵坐标代表原始有限差分 法算得对应位置的格林函数表表项. 最终验证这 些表项具有完全相同的数值, 具有相同的准确度.

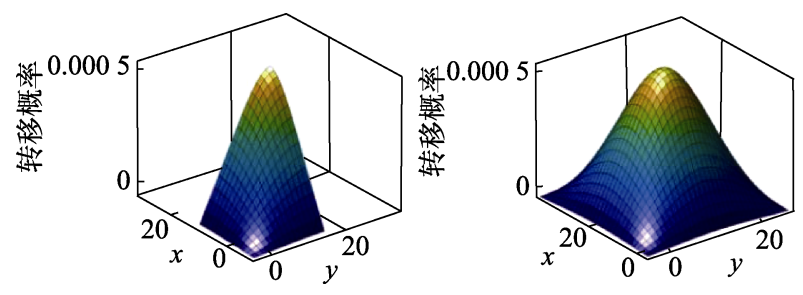

图 5 原始和改进有限差分法格林函数表比较

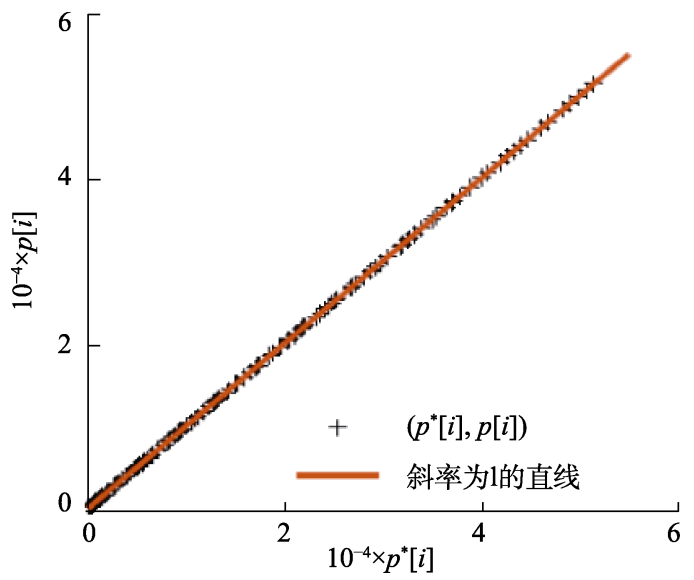

图 6 原始和改进有限差分法格林函数表项比较

(1) http://www.openblas.net 


\section{4 结 语}

本文证明并利用了多介质格林函数表的对称 性，提出了一种多介质格林函数表的高效生成方 法. 数值实验表明, 该方法相比于原始的有限差分 方法，在保证生成完全相同结果的基础上，将所需 时间开销降低到了原来耗时的 $1 / 8$ 以下. 改进的生 成方法显著地提高了悬浮随机行走电容提取预刻 画的效率。

\section{参考文献(References):}

[1] Yu W J, Wang X R. Advanced field-solver techniques for RC extraction of integrated circuits[M]. Heidelberg: Springer, 2014

[2] Le Coz Y L, Iverson R B. A stochastic algorithm for high speed capacitance extraction in integrated circuits[J]. Solid-State Electronics, 1992, 35(7): 1005-1012

[3] Yu W J, Zhuang H, Zhang C, et al. RWCap: a floating random walk solver for 3-D capacitance extraction of very-large-scale integration interconnects[J]. IEEE Transactions on Computer-Aided Design of Integrated Circuits and Systems, 2013, 32(3): 353-366

[4] Dillinger T. Field-solver parasitic extraction goes mainstream[OL]. [2020-07-22]. https://www.semiwiki.com/forum/ content/7154-field-solver-parasiticextraction-goes-mainstream. html

[5] RWCap[OL]. [2020-07-22] http://numbda.cs.tsinghua.edu.cn/ download/RWCap_v3_en.html

[6] Zhang C, Yu W J. Efficient techniques for the capacitance extraction of chip-scale VLSI interconnects using floating random walk algorithm[C] //Proceedings of the 19th Asia South Pacific Design Automation Conference. Los Alamitos: IEEE Computer Society Press, 2014: 756-761
[7] Zhang B L, Yu W J, Zhang C. Improved pre-characterization method for the random walk based capacitance extraction of multi-dielectric VLSI interconnects[J]. International Journal of Numerical Modelling: Electronic Networks, Devices and Fields, 2016, 29(1): 21-34

[8] Zhang C, Yu W J, Wang Q, et al. Fast random walk based capacitance extraction for the 3-D IC structures with cylindrical inter-tier-vias[J]. IEEE Transactions on Computer-Aided Design of Integrated Circuits and Systems, 2015, 34(12): 1977-1990

[9] Xu Z Z, Zhang C, Yu W J. Floating random walk-based capacitance extraction for general non-Manhattan conductor structures[J]. IEEE Transactions on Computer-Aided Design of Integrated Circuits and Systems, 2017, 36(1): 120-133

[10] Yu W J, Xu Z Z, Li B, et al. Floating random walk-based capacitance simulation considering general floating metals[J]. IEEE Transactions on Computer-Aided Design of Integrated Circuits and Systems, 2018, 37(8): 1711-1715

[11] Zhai K Y, Yu W J, Zhuang H. GPU-friendly floating random walk algorithm for capacitance extraction of VLSI inter-connects[C] //Proceedings of the Conference on Design, Automation and Test in Europe. Los Alamitos: IEEE Computer Society Press, 2013:1661-1666

[12] Wei X, Yan C H, Zhou H, et al. An efficient FPGA-based floating random walk solver for capacitance extraction using SDAccel[C] //Proceedings of the Design, Automation \& Test in Europe Conference \& Exhibition. Los Alamitos: IEEE Computer Society Press, 2019: 1040-1045

[13] Xu Z Z, Yu W J, Zhang C, et al. A parallel random walk solver for the capacitance calculation problem in touch- screen design[C] //Proceedings of the International Great Lakes Symposium on VLSI. Los Alamitos: IEEE Computer Society Press, 2016: 99-104

[14] Boundary condition[OL]. [2020-07-22]. https://mathworld. wolfram.com/BoundaryConditions.html

[15] SuiteSparse[OL]. [2020-07-22]. faculty.cse.tamu.edu/davis/ suitesparse.html 